\title{
Brain Structural Changes in Regions Within the Salience Network in Patients with Functional Constipation
}

\section{Zhenzhen Jia}

Xidian University

Guanya Li

Xidian University

Yang Hu

Xidian University

Hao Li

Xidian University

Wenchao Zhang

Xidian University

Jia Wang

Xidian University

Lei Zhang

Xidian University

Zongxin Tan

Xidian University

Shuai Lv

Xidian University

Karen M. von Deneen

Xidian University

\section{Shijun Duan}

Air Force Medical University Tangdu Hospital

Guangbin Cui

Air Force Medical University Tangdu Hospital

Yi Zhang ( $\square$ zyrisk@163.com )

Xidian University https://orcid.org/0000-0003-0859-9735

Yongzhan Nie

Air Force Medical University Xijing Hospital: Xijing Hospital 
Keywords: functional constipation, diffusion tensor imaging, voxel-based morphometry, gray matter volume, salience network

Posted Date: December 3rd, 2021

DOI: https://doi.org/10.21203/rs.3.rs-1105989/v1

License: (1) This work is licensed under a Creative Commons Attribution 4.0 International License. Read Full License

Version of Record: A version of this preprint was published at Brain Imaging and Behavior on March 12th, 2022. See the published version at https://doi.org/10.1007/s11682-022-00648-3. 


\section{Abstract}

Functional constipation (FCon) is one of the common functional gastrointestinal disorders (FGID). Previous studies reported alterations in cortical morphometry as well as changes in white matter (WM) fiber tracts and thalamo-limbic/parietal structural connectivity (SC). However, whether patients with FCon are implicated in changes in gray matter (GM) volume and associated SC remains unclear. Voxel-based morphometry (VBM) was first employed to examine differences in GM volume between 48 patients with FCon and 52 healthy controls (HC). Diffusion tensor imaging (DTI) with probabilistic tractography analysis was then employed to explore alterations in SC of these regions. Compared with the HC, patients with FCon showed decreased GM volumes in the right middle frontal gyrus (MFG_R), left insula (INS_L), and anterior cingulate cortex $\left(\mathrm{ACC}, P_{\mathrm{FWE}}<0.05\right)$ which had a negative correlation with abdominal symptoms and difficulty of defecation respectively. Seed-based SC showed patients with FCon had decreased fractional anisotropy (FA) of bilateral INS-ACC, bilateral ACC-MFG, bilateral INS-MFG, increased axial diffusivity (AD) of bilateral ACC-MFG, and decreased radial diffusivity (RD) of bilateral INS-ACC, bilateral ACC-MFG tracts. FA of the right INS-ACC tract had a negative correlation with difficulty of defecation and AD of the ACC-left MFG tract had a negative correlation with stool symptoms. These findings reflect patients with FCon are implicated in changes in GM volumes and corresponding SC in brain regions within the salience network.

\section{Introduction}

Functional constipation (FCon) is one of the common functional gastrointestinal disorders (FGID) (Alame and Bahna 2012; De Giorgio et al. 2015; Koppen et al. 2015) with an average female-male ratio of 2.1:1 (Mugie et al. 2010) and a prevalence ranging from 0.7-79\% in clinical practice (Alame and Bahna 2012; Koppen et al. 2015; Mugie et al. 2010). FCon features infrequent bowel movements, difficulty of defecation, large and/or hard stools, excessive straining, and is usually associated with abdominal distension/pain (Alame and Bahna 2012; De Giorgio et al. 2015; Koppen et al. 2015). These symptoms have imposed a huge burden on families and society because of impaired quality of life (Bongers et al. 2009) and emotional status (Jones et al. 2006).

In recent years, neuroimaging has been gradually employed to assess brain structural and functional changes in patients with FCon (Li et al. 2021; Blankstein et al. 2010; Hu et al. 2020; Jin et al. 2019; Mayer et al. 2009; Tillisch et al. 2011). Previous resting-state functional magnetic resonance imaging (RS-fMRI) report showed FCon related brain functional abnormalities were in areas associated with emotional modulation including anterior insula-alNS, hippocampal gyrus-HIPP, orbital frontal cortex-OFC, and dorsal anterior cingulate cortex-dACC (Zhu et al. 2016). Another RS-fMRI report was designed to examine gender-related differences in resting-state brain activity and functional connectivity in FCon patients, and results showed lower basal brain activities (i.e., insula-INS, precentral gyrus-PreCen, thalamus, and OFC) in female FCon patients than in males and INS/OFC had a negative correlation with FCon symptoms (Jin et al. 2019). One study combining graph theory with RS-fMRI showed that FCon had small-worldness and a low normalized clustering coefficient, indicating reduced brain functional connectivity, which were 
predominately related to the rostral anterior cingulate cortex (emotional processing), SMA (motor-control), and thalamus (somatic/sensory) (Liu et al. 2021). One newly published study reported FCon showed decreased activity in fractional amplitude of low-frequency fluctuation in the perigenual anterior cingulate cortex, dorsomedial prefrontal cortex and precuneus (Li et al. 2021).

Growing evidence have showed that brain functional abnormalities might be associated with structural changes (Hao et al. 2013; Hubbard et al. 2016; Wang et al. 2020). Previous MRI study reported cortical morphometric alterations in brain areas involved with emotional processing (middle frontal cortex-MFG, $\mathrm{dACC}$, and OFC), self-referential processing (precuneus/posterior cingulate cortex), and somatic/motorcontrol (SMA) in FCon group (Hu et al. 2020). Cortical morphological abnormalities may be related to changes in diffusion parameters (Tamnes et al. 2010). Another recent study showed that FCon was related to changes in thalamo-limbic/parietal structural connectivity (SC) (Zhang et al. 2020). However, few studies have been performed to examine the alterations in gray matter (GM) volume and associated $\mathrm{SC}$ in patients with FCon.

In addition, one structural MRI study reported that IBS patients displayed significant alterations in GM volumes in the middle frontal gyrus (MFG), anterior cingulate cortex (ACC), INS, and HIPP (Zhao et al. 2018). Diffusion tensor imaging (DTI) detected abnormalities in SC between INS and ACC in IBS patients (Chen et al. 2011). Female IBS patients had decreased GM volumes in the INS, MFG, ACC, and HIPP (Labus et al. 2014). These previous brain imaging studies on IBS consistently recorded changes in brain regions implicated in emotional processing and modulation, especially in the aINS and ACC, which are key nodes of the salience network (SN) (Chang et al. 2013; Garcia-Larrea 2012; Mayer et al. 2015). The SN, which includes INS, ACC, and MFG, is responsible for integrating all kinds of sensory information and directing specific brain regions to respond appropriately (Peters et al. 2016). However, whether FCon is implicated in structural abnormalities in the $\mathrm{SN}$ has not been investigated yet.

The current study was designed to explore the brain structural changes in FCon patients. Thus, voxelbased morphometry (VBM) was first employed to explore differences in GM volumes between 48 FCon patients and 52 healthy controls $(\mathrm{HC})$, and regions showing significant differences were then selected as seed regions for probabilistic tractography to feature regional SC as quantified by fractional anisotropy $(F A)$, mean- (MD), axial- $(A D)$, and radial diffusivity (RD). The study aimed to test whether: (a) FCon would be associated with abnormality in GM volumes in brain regions within the $\mathrm{SN}$, which are implicated in interoception and emotional processing. (b) FCon would be associated with alterations in SC of brain regions within the SN.

\section{Materials And Methods}

\section{Participants}

The experimental protocol was approved by the Institutional Review Board of Xijing Hospital and registered in the Chinese Clinical Trial Registry Center as: ChiCTR-OOB-15006347 
(http://www.chictr.org.cn). The experiments were conducted in accordance with the Declaration of Helsinki. Fifty-four patients with FCon were recruited from a clinical site at Xijing Hospital affiliated with the Air Force Medical University in Xi'an, China. Fifty-two HC subjects were recruited from the local community. All participants in the experiment were right-handed. They signed an informed consent form as well as performed a full physical examination with history. FCon was diagnosed using Rome IV criteria through a gastroenterologist experienced in FGID diagnosis (Drossman 2016). FCon subjects with various main bowel habits such as functional defecatory disorders, slow transit constipation, and a combination of the two types were included in the experiment and constipation after childbirth, congenital giant colon/redundant sigmoid colon/pelvic floor muscle relaxation, psychiatric/medical/neurological disorders demanding instant treatment, and existing medicine taken that might influence the central nervous system were removed. Four females and two males with dental implants were excluded from the experiment. Then, the study included fifty-two HC subjects and fortyeight patients with FCon. All subjects were demanded to finish several self-administered questionnaires, such as difficulty of defecation and Patient Assessment of Constipation Symptoms Questionnaire which consists of abdominal symptoms, rectal symptoms, and stool symptoms (Frank et al. 1999). All subjects finished the ZUNG self-rating anxiety scale (SAS) (Zung 1971) and ZUNG self-rating depressive scale (SDS) (ZUNG 1965) to estimate the severity of their anxiety/depression (Table 1). The study excluded all subjects with anxiety/depression.

Table 1

Demographic and clinical information of $\mathrm{HC}$ and FCon groups

\begin{tabular}{|llll|}
\hline & HC (N = 52) & FCon (N = 48) & HC vs. FCon \\
\hline Age (years) & $($ Mean \pm SE $)$ & $($ Mean \pm SE $)$ & $P$ value \\
\hline Gender & $38.0 \pm 2.0$ & $43.4 \pm 1.8$ & 0.052 \\
\hline Depression (SDS) (0-80) & $34 \mathrm{~F} / 18 \mathrm{M}$ & $37 \mathrm{~F} / 11 \mathrm{M}$ & 0.198 \\
\hline Anxiety (SAS) (0-80) & $40.8 \pm 1.4$ & $43.2 \pm 1.1$ & 0.167 \\
\hline Difficulty of defecation (0-100) & $36.6 \pm 1.0$ & $39.0 \pm 0.9$ & 0.074 \\
\hline Stool symptoms & N/A & $73.5 \pm 2.6$ & N/A \\
\hline Rectal symptoms & N/A & $3.7 \pm 0.3$ & N/A \\
\hline Abdominal symptoms & N/A & $3.7 \pm 0.3$ & N/A \\
\hline $\begin{array}{l}\text { Abbreviation: HC, healthy controls; FCon, functional constipation; SDS, ZUNG Self-rating Depressive } \\
\text { Scale; SAS, ZUNG Self-rating Anxiety Scale; SE, standard error. }\end{array}$ & N/A \\
\hline
\end{tabular}

MRI acquisition 
MRI scans were implemented between 9 A.M. and 10 A.M. to guarantee uniformity of judgments and to minimize diurnal variation. The experiment of the study was based on a 1.5 T Signa HDXT scanner (GE, Milwaukee, WI, USA). Firstly, the high-resolution structural images of all subjects were obtained by threedimensional magnetization-prepared fast acquisition gradient-echo sequences with a voxel size of 1 $\mathrm{mm}^{3}$ as well as an axial fast-spoiled-gradient-echo sequence (matrix size $=256 \times 256, \mathrm{TR}=9.1 \mathrm{~ms}, \mathrm{TE}=$ $3.0 \mathrm{~ms}$, slice thickness $=1 \mathrm{~mm}$ as well as 248 slices, and field-of-view $=512 \times 512 \mathrm{~mm}^{2}$ ). Secondly, diffusion-weighted (DW) images (single shot spin echo planar imaging sequence) with 1 non-diffusion weighting $\left(b=0 \mathrm{~s} / \mathrm{mm}^{2}\right)$ and 25 non-collinear diffusion sensitizing directions $\left(b=1000 \mathrm{~s} / \mathrm{mm}^{2}\right)$ were obtained, and the parameters were: matrix size $=128 \times 128, \mathrm{TR}=8000 \mathrm{~ms}, \mathrm{TE}=98.3 \mathrm{~ms}$, field-of-view $=$ $256 \times 256 \mathrm{~mm}^{2}, 30$ continuous axial slices, and slice thickness $=5 \mathrm{~mm}$.

\section{Voxel-based morphometry of gray and white matter volumes}

The 3D structural images were analyzed by VBM toolbox (http://dbm.neuro.uni-jena.de/vbm/download/) and Statistical Parametric Mapping (SPM 12, https://www.fil.ion.ucl.ac.uk/spm/). Preprocessing steps containing realignment, bias-correction, tissue classification, and spatial normalization were implemented on the two groups respectively.

\section{Statistical parametric mapping (identification of region of interest)}

Voxel-wise analysis on GM brain mappings was implemented using SPM 12. Between HC and FCon groups, they were examined by a two-sample $t$-test with age/gender regressed out as covariates. Prominent GM volumes were chosen as regions of interest (ROIs) after whole brain family wise error (FWE) correction $(P<0.05)$ at the minimum cluster size of 30 voxel level. Contralateral brain areas were also identified as ROIs. Five ROIs were selected for fiber tracking using Subcortical Structural probabilistic atlases (Desikan et al. 2006) and FSL's Harvard-Oxford Cortical and AAL atlas (Tzourio-Mazoyer et al. 2002) including: ACC, right insula (INS_R), left insula (INS_L), right middle frontal gyrus (MFG_R), and left middle frontal gyrus (MFG_L).

\section{DW-MRI tractography analysis}

FMRIB's Diffusion Toolbox (FDT), which is embedded in FMRIB Software Library (FSL) version 5.0.9 software package (http://www.fmrib.ox.ac.uk/fsl/), was employed to pretreatment DTI information as well as implement probabilistic tractography. Firstly, FSL Brain Extractor Tool (Smith 2002) was employed to eliminate non-brain tissue. Eddy-current correction and head motion were conducted by FDT in the FSL. Secondly, Diffusion Tensor Imaging Fit (DTIFIT) in FDT was employed to fit a diffusion tensor model at each voxel of the pretreated eddy current corrected diffusion-weighted data and to obtain the FA, $M D, A D$, and RD maps. Then, Bayesian Estimation of Diffusion Parameters Obtained using Sampling Technique was used to evaluate the fiber direction distribution at every brain voxel (Hirsiger et al. 2016). 
Probabilistic fiber tractography was calculated for every participants by probtrackx 2 to produce the most likely connectivity distribution between ROI pairs. Tractography parameters were: 5000 samples per seed voxel, 0.2 curvature threshold, subsidiary fiber volume threshold of 0.01 , step length of $0.5 \mathrm{~mm}$, and a maximum number of steps 2000 . Final-tract mask was obtained by overlapping the i-tract and j-tract, which respectively represent a seed mask and a target mask. The mean value of FA/MD/AD/RD within the final-tract mask connecting each ROI pair was calculated (Hirsiger et al. 2016). Participants who failed tractography/ROI conversion were removed from the data analysis (resulting in $2 \mathrm{HC}$ subjects and 3 patients with FCon exclusions) (Hirsiger et al. 2016).

\section{Statistical analysis}

Demographic and clinical variables were analyzed by SPSS Statistics (Version 22, IBM). Chi-square test was employed to evaluate differences in gender distribution. Two sample $t$-test was employed to examine the discrepancies in behavioral measurements. Diffusion parameters were employed to assess the degree of connectivity for every ROI pair by regressing out age and gender $(P<0.05)$.

\section{Association between behaviors and GM/DTI}

Partial correlation analysis with age and gender as covariates was conducted to evaluate the correlations among GM volumes, diffusion parameters, and clinical variables. Bonferroni correction was used for multiple comparisons, and significance level was set at $P<0.0028(0.05 / 18)$ between $\mathrm{GM}$ volumes and clinical variables, and significance level was set at $P<0.0014(0.05 / 36)$ between diffusion parameters and clinical variables.

\section{Results}

\section{Demographic characteristics}

The study included forty-eight patients with FCon (age $43.4 \pm 1.8$ years, range 20 - 50 years, 11 males) and fifty-two HC subjects (age, education, gender, SDS, and SAS matched, $P>0.05$; age $38.0 \pm 2.0$ years, range 20 - 50 years, 18 males) (Table 1 ). Table 1 showed difficulty of defecation, stool symptoms, rectal symptoms, and abdominal symptoms in FCon group.

\section{Alterations in $\mathrm{GM}$ volumes}

FCon group showed significant decreases in GM volumes in INS_L, ACC, and MFG_R than HC $\left(P_{\mathrm{FWE}}<\right.$ 0.05, Fig. 1A, Fig. 1B).

GM volume in the INS_L had a negative correlation with abdominal symptoms $(r=-0.51, P=0.0003)$, and GM volume in the ACC had a negative correlation with difficulty of defecation in the FCon group $(r=-0.46$, $P=0.0011$, Fig. 1C).

\section{Alterations in SC}


DTI with probabilistic tractography results showed the final-tract changes between INS and ACC, between ACC and MFG, and between INS and MFG (Fig. 2-3) (Table 2). FCon group showed significant decreased FA of the left INS-ACC, right INS-ACC, ACC-left MFG, ACC-right MFG, left INS-left MFG, and right INS-right MFG tracts than $\mathrm{HC}(P<0.05$, Fig. 2). FCon group also showed significant decreased $A D$ of the ACC-left MFG and ACC-right MFG tracts and increased RD of the left INS-ACC, right INS-ACC, ACC-left MFG, and ACC-right MFG tracts $(P<0.05$, Fig. 3$)$.

Table 2

Differences in diffusion parameters in $\mathrm{HC}$ and FCon groups

\begin{tabular}{|c|c|c|c|c|}
\hline \multirow[t]{2}{*}{$\begin{array}{l}\text { Fiber tracts between } \\
\text { ROI pairs }\end{array}$} & \multirow[t]{2}{*}{$\begin{array}{l}\text { Diffusion } \\
\text { parameters }\end{array}$} & $\mathrm{HC}(\mathrm{N}=50)$ & FCon $(N=45)$ & $\begin{array}{l}\text { HC vs. } \\
\text { FCon }\end{array}$ \\
\hline & & $($ Mean \pm SE ) & (Mean \pm SE ) & $P$ value \\
\hline \multirow[t]{2}{*}{ INS_L - ACC } & FA & $0.350 \pm 0.004$ & $0.335 \pm 0.003$ & 0.024 \\
\hline & $\mathrm{RD}$ & $\begin{array}{l}6.81 \times 10^{-4} \pm 5.02 \\
\times 10^{-6}\end{array}$ & $\begin{array}{l}7.00 \times 10^{-4} \pm 5.43 \\
\times 10^{-6}\end{array}$ & 0.028 \\
\hline \multirow[t]{2}{*}{ INS_R - ACC } & FA & $0.346 \pm 0.004$ & $0.331 \pm 0.003$ & 0.012 \\
\hline & $\mathrm{RD}$ & $\begin{array}{l}6.95 \times 10^{-4} \pm 5.39 \\
\times 10^{-6}\end{array}$ & $\begin{array}{l}7.15 \times 10^{-4} \pm 6.13 \\
\times 10^{-6}\end{array}$ & 0.024 \\
\hline \multirow[t]{3}{*}{ MFG_L - ACC } & $A D$ & $\begin{array}{l}1.24 \times 10^{-3} \pm 5.08 \\
\times 10^{-6}\end{array}$ & $\begin{array}{l}1.23 \times 10^{-3} \pm 4.62 \\
\times 10^{-6}\end{array}$ & 0.015 \\
\hline & FA & $0.381 \pm 0.003$ & $0.361 \pm 0.004$ & 0.002 \\
\hline & $\mathrm{RD}$ & $\begin{array}{l}6.79 \times 10^{-4} \pm 4.65 \\
\times 10^{-6}\end{array}$ & $\begin{array}{l}7.01 \times 10^{-4} \pm 6.13 \\
\times 10^{-6}\end{array}$ & 0.023 \\
\hline \multirow[t]{3}{*}{ MFG_R - ACC } & $A D$ & $\begin{array}{l}1.25 \times 10^{-3} \pm 5.45 \\
\times 10^{-6}\end{array}$ & $\begin{array}{l}1.22 \times 10^{-3} \pm 5.27 \\
\times 10^{-6}\end{array}$ & 0.019 \\
\hline & FA & $0.388 \pm 0.004$ & $0.362 \pm 0.004$ & $<0.001$ \\
\hline & $\mathrm{RD}$ & $\begin{array}{l}6.73 \times 10^{-4} \pm 5.65 \\
\times 10^{-6}\end{array}$ & $\begin{array}{l}7.00 \times 10^{-4} \pm 5.09 \\
\times 10^{-6}\end{array}$ & 0.003 \\
\hline INS_L - MFG_L & FA & $0.348 \pm 0.003$ & $0.328 \pm 0.003$ & $<0.001$ \\
\hline INS_R - MFG_R & FA & $0.342 \pm 0.003$ & $0.322 \pm 0.003$ & $<0.001$ \\
\hline
\end{tabular}

FA of the right INS-ACC tract had a negative correlation with difficulty of defecation $(r=-0.48, P=0.0008$, Fig. 2), and AD of the ACC-left MFG tract had a negative correlation with stool symptoms $(r=-0.52, P=$ 


\section{Discussion}

VBM and DTI with probabilistic tractography analysis were employed to explore differences in brain GM volume and SC between FCon and HC groups. FCon group had significant decreases in GM volumes in the INS, ACC, and MFG involved in interoceptive and emotional processing. GM volume in the INS had a negative correlation with abdominal symptoms, and GM volume in the ACC had a negative correlation with difficulty of defecation in the FCon group. Results also showed significant decreased FA and increased RD of bilateral INS-ACC, decreased AD/FA, and increased RD of the bilateral ACC-MFG, and decreased FA of the bilateral INS-MFG tracts in FCon group. FA of the right INS-ACC tract had a negative correlation with difficulty of defecation and AD of the ACC-left MFG tract had a negative correlation with stool symptoms.

\section{Alterations in GM volume within SN}

Results showed FCon group had decreased GM volumes in the INS, ACC, and MFG, which are key nodes of the SN (Peters et al. 2016). SN is essential for the integration of visceral and sensory information (Peters et al. 2016; Uddin 2015).

Patients with FCon had decreased GM volume in the INS, which is consistent with changes in patients with IBS (Labus et al. 2014; Zhao et al. 2018). The INS is responsible for interoceptive information processing related to the organism, and plays a crucial role in integrating visceral sensation, emotional processing, and high-grade cognition (Craig 2002; Critchley et al. 2004; Garcia-Larrea 2012; Mayer et al. 2009; Starr et al. 2009; Uddin et al. 2017). A previous study reported that electrocortical stimulation of the INS caused visceral sensory responses, including abdominal sensation, stomach vibration, and vomiting (Stephani et al. 2011). Reduced GM volume in INS in FCon group might disrupt the internal information perception. Data showed the negative association between abdominal symptoms and GM volume in the INS in FCon group, reflecting the role of the INS in sensing visceral pain (Keszthelyi et al. 2012). One RSfMRI reported that FCon showed abnormal baseline brain activities in aINS and amplitude of low frequency fluctuation (ALFF) in alNS had a negative correlation with difficulty of defecation (Zhu et al. 2016). Another RS-fMRI study reported that FCon showed gender effects on baseline brain activity in the INS, and that ALFF in the INS had a positive correlation with abdominal pain (Blankstein et al. 2010). A previous IBS study also reported that decreased excitatory neurotransmitter grades in alNS were related to abdominal pain (Bednarska et al. 2019). These previous studies indicate that INS is related to interoceptive processing of visceral sensation including abdominal pain and/or distension, and decreased GM volume in the INS might account for its functional abnormality reported in patients with IBS and FCon.

FCon group had also decreased GM volumes in areas related to emotional processing (ACC, MFG). The ACC is primarily concerned with executive-control of internal/external stimuli, affective responses to 
visceral sensation, and modulation of emotional responses (George et al. 2000). The MFG is the additional cortical node of the SN (Peters et al. 2016), which is critically implicated in visceral sensory information, emotional processing, and cognitive-control (Hu et al. 2020; Liu et al. 2021). One study reported that FCon displayed reduced cortical thickness in the MFG and dACC, and duration of constipation had a negative correlation with cortical thickness in the ACC (Hu et al. 2020). FCon also showed abnormal baseline brain activities in the ACC (Zhu et al. 2016). One MRI study on IBS revealed abnormalities in the ACC during rectal stimulation (Wang et al. 2017). MFG was related to attention control and chronic pain modulation (Lorenz et al. 2003). Data showed that there was no significant association between the MFG and constipation symptoms, which might be related to the small sample size (controlling for age, gender, SAS, and SDS). However, in FCon group, GM volume in ACC had a negative association with difficulty of defecation. These findings suggested that constipation symptoms such as difficulty of defecation might not only be associated with abnormalities in function and cortical morphology, but also with changes in GM volumes in regions involved with emotional processing, highlighting the critical role of the ACC in FCon.

\section{Alterations in SC within SN}

Previous studies reported abnormal brain activation in the INS, ACC, MFG, thalamus, OFC, HIPP, and PreCen during visceral stimulation in patients with FCon (Hu et al. 2020; Jin et al. 2019; Liu et al. 2021; Zhang et al. 2020; Zhu et al. 2016). One recent study using DTI with probabilistic tractography reported FCon was implicated in changes in the SC of thalamo-limbic/parietal circuits (Zhang et al. 2020). In the current study, alterations in the SC within SN were assessed by FA/MD/AD/RD, indicating that WM fiber tracts make a valuable contribution towards patients with FCon (Ellingson et al. 2013; Fang et al. 2017).

Results showed decreased FA and increased RD of the bilateral INS-ACC tract, and FA in the right INS-ACC tract had a negative correlation with difficulty of defecation. The findings indicated that the better the integrity of WM, the less severe the constipation symptoms such as difficulty of defecation. One RS-fMRI research about FCon showed increased ALFF in aINS and AACC and stronger causal influence from dACC to alNS (Zhu et al. 2016), which are consistent with our SC results, suggesting the structural alterations might underlie changes in functional connectivity.

Results also showed decreased FA/AD and increased RD of the bilateral ACC-MFG tract, and AD in the left ACC-MFG tract had a negative correlation with stool symptoms. The findings suggested that the better the integrity of WM, the less severe the stool symptoms. Previous fMRI studies reported that the ACC and MFG were implicated in emotional regulation processing (Beauregard et al. 2001; Hu et al. 2020; Zhu et al. 2016). One cortical morphometric study reported that FCon displayed reduced cortical thickness in the dACC and MFG (Hu et al. 2020). Another study combining DTI and RS-fMRI reported that patients with borderline (Koppen et al. 2015) personality disorder showed abnormal SC and functional connectivity between the ACC and MFG (Lei et al. 2019). Thus, our data indicated that abnormal SC between the ACC and MFG might be associated with dysfunction in emotional regulation processing in patients with FCon. 
The current study also showed decreased FA of the bilateral INS-MFG tracts. A previous RS-MRI report revealed that FCon patients showed increased ALFF in the aINS (Zhu et al. 2016). Another study reported FCon displayed reduced cortical thickness in the MFG (Hu et al. 2020). These findings suggested that abnormal SC between the INS and MFG is associated with emotional processing of visceral sensations.

\section{Conclusion}

The study was designed to examine brain GM volume and corresponding SC changes in FCon patients. Results showed that FCon group had lower GM volumes in the ACC, MFG, and INS than HC. GM volume in the INS had a negative correlation with abdominal symptoms, and GM volume in the ACC had a negative correlation with difficulty of defecation. Results also showed decreased FA and increased RD of the bilateral INS-ACC, decreased AD/FA and increased RD of the bilateral ACC-MFG, and decreased FA of the bilateral INS-MFG tracts in FCon group. FA of the right INS-ACC tract had a negative correlation with difficulty of defecation. AD of the ACC-left MFG tract had a negative correlation with stool symptoms. These discoveries reflect that FCon is involved in changes in GM volumes and corresponding SC in brain regions within the salience network.

\section{Declarations}

\section{Conflict of Interest}

The authors declare no conflict of interest.

\section{Ethical statements}

Informed consent was obtained from all patients included in the study.

\section{Ethical approval}

All procedures performed in studies involving human participants were in accordance with the ethical standards of the institutional and/or national research committee and with the 1964 Helsinki Declaration and its later amendments or comparable ethical standards.

\section{Acknowledgements}

We thank all participants and their families for participating in our study. This work was supported by the National Natural Science Foundation of China under Grant Nos. 82172023, 81470816, 81730016; the Open Funding Project of National Key Laboratory of Human Factors Engineering, Grant No. 6142222190103; and National Clinical Research Center for Digestive Diseases, Xi'an, China under Grant No. 2015BAl13B07.

\section{Authors' contribution}


Study concept and design ( $\mathrm{YN}, \mathrm{YZ}$, and $\mathrm{GL}$ ), diagnosis ( $\mathrm{YN}, \mathrm{ZJ}$, and $\mathrm{YZ}$ ), image data collection (GC, SD, and $Z \mathrm{~J}$ ), data analysis (ZJ, GL, $Y H$, and $\mathrm{HL}$ ), data interpretation (KMV and $Y Z$ ), writing of manuscript (ZJ and $Y Z$ ), and review and approval of manuscript (all authors).

\section{References}

1. Alame, A., \& Bahna, H. (2012). Evaluation of Constipation. Clinics in Colon and Rectal Surgery, 25(01), 5-11

2. Beauregard, M., Levesque, J., \& Bourgouin, P. (2001). Neural Correlates of Conscious Self-Regulation of Emotion. The Journal of neuroscience, 21(18), 165

3. Bednarska, O., Icenhour, A., Tapper, S., Witt, S. T., Tisell, A., Lundberg, P., et al. (2019). Reduced excitatory neurotransmitter levels in anterior insulae are associated with abdominal pain in irritable bowel syndrome. Pain, Publish Ahead of Print

4. Blankstein, U., Chen, J., Diamant, N. E., \& Davis, K. D. (2010). Altered Brain Structure in Irritable Bowel Syndrome: Potential Contributions of Pre-Existing and Disease-Driven Factors. Gastroenterology, 138(5), 1783-1789

5. Bongers, M. E. J., van Dijk, M., Benninga, M. A., \& Grootenhuis, M. A. (2009). Health Related Quality of Life in Children with Constipation-Associated Fecal Incontinence. The Journal of Pediatrics, 154(5), $749-753$

6. Chang, L. J., Yarkoni, T., Khaw, M. W., \& Sanfey, A. G. (2013). Decoding the Role of the Insula in Human Cognition: Functional Parcellation and Large-Scale Reverse Inference. Cerebral Cortex, 23(3), $739-749$

7. Chen, J. Y., Blankstein, U., Diamant, N. E., \& Davis, K. D. (2011). White matter abnormalities in irritable bowel syndrome and relation to individual factors. Brain Research, 1392, 121-131

8. Craig, A. D. (2002). How do you feel? Interoception: the sense of the physiological condition of the body. Nat Rev Neurosci, 3(8), 655-666

9. Critchley, H. D., Wiens, S., Rotshtein, P., Öhman, A., \& Dolan, R. J. (2004). Neural systems supporting interoceptive awareness. Nature Neuroscience, 7(2), 189-195

10. De Giorgio, R., Ruggeri, E., Stanghellini, V., Eusebi, L. H., Bazzoli, F., \& Chiarioni, G. (2015). Chronic constipation in the elderly: a primer for the gastroenterologist. BMC gastroenterology, 15(1), 130

11. Desikan, R. S., Ségonne, F., Fischl, B., Quinn, B. T., Dickerson, B. C., Blacker, D., et al. (2006). An automated labeling system for subdividing the human cerebral cortex on MRI scans into gyral based regions of interest. Neurolmage, 31(3), 968-980

12. Drossman, D. A. (2016). Functional Gastrointestinal Disorders: History, Pathophysiology, Clinical Features, and Rome IV. Gastroenterology, 150(6), 1262-1279

13. Ellingson, B. M., Mayer, E., Harris, R. J., Ashe-McNally, C., Naliboff, B. D., Labus, J. S., et al. (2013). Diffusion tensor imaging detects microstructural reorganization in the brain associated with chronic irritable bowel syndrome. Pain, 154(9), 1528-1541 
14. Fang, J., Li, S., Li, M., Chan, Q., Ma, X., Su, H., et al. (2017). Altered white matter microstructure identified with tract-based spatial statistics in irritable bowel syndrome: a diffusion tensor imaging study. Brain Imaging and Behavior, 11(4), 1110-1116

15. Frank, L., Kleinman, L., Farup, C., Taylor, L., \& Miner, P. J. (1999). Psychometric validation of a constipation symptom assessment questionnaire. Scand J Gastroenterol, 34(9), 870-877

16. Garcia-Larrea, L. (2012). The posterior insular-opercular region and the search of a primary cortex for pain. Neurophysiol Clin, 42(5), 299-313

17. George, B., Phan, L., \& Michael, I. P. (2000). Cognitive and emotional influences in anterior cingulate cortex. Trends in Cognitive Sciences, $4(6)$

18. Hao, X., Xu, D., Bansal, R., Dong, Z., Liu, J., Wang, Z., et al. (2013). Multimodal magnetic resonance imaging: The coordinated use of multiple, mutually informative probes to understand brain structure and function. Human Brain Mapping, 34(2), 253-271

19. Hirsiger, S., Koppelmans, V., Mérillat, S., Liem, F., Erdeniz, B., Seidler, R. D., et al. (2016). Structural and functional connectivity in healthy aging: Associations for cognition and motor behavior. Human Brain Mapping, 37(3), 855-867

20. Hu, C., Liu, L., Liu, L., Zhang, J., Hu, Y., Zhang, W., et al. (2020). Cortical morphometry alterations in brain regions involved in emotional, motor-control and self-referential processing in patients with functional constipation. Brain Imaging and Behavior, 14(5), 1899-1907

21. Hubbard, C. S., Becerra, L., Heinz, N., Ludwick, A., Rasooly, T., Wu, R., et al. (2016). Abdominal Pain, the Adolescent and Altered Brain Structure and Function. PloS one, 11(5), e156545

22. Jin, Q., Duan, S., Li, G., Sun, L., Hu, Y., Hu, C., et al. (2019). Sex-related differences in resting-state brain activity and connectivity in the orbital frontal cortex and insula in patients with functional constipation. Neurogastroenterology \& Motility, 31(5), e13566

23. Jones, M. P., Dilley, J. B., Drossman, D., \& Crowell, M. D. (2006). Brain-gut connections in functional GI disorders: anatomic and physiologic relationships. Neurogastroenterology and Motility, 18(2), 91103

24. Keszthelyi, D., Troost, F. J., \& Masclee, A. A. (2012). Irritable Bowel Syndrome: Methods, Mechanisms, and Pathophysiology. Methods to assess visceral hypersensitivity in irritable bowel syndrome. American Journal of Physiology-Gastrointestinal and Liver Physiology, 303(2), G141-G154

25. Koppen, I. J. N., Lammers, L. A., Benninga, M. A., \& Tabbers, M. M. (2015). Management of Functional Constipation in Children: Therapy in Practice. Paediatric drugs, 17(5), 349-360

26. Labus, J. S., Dinov, I. D., Jiang, Z., Ashe-McNalley, C., Zamanyan, A., Shi, Y., et al. (2014). Irritable bowel syndrome in female patients is associated with alterations in structural brain networks. Pain, 155(1), 137-149

27. Lei, X., Zhong, M., Zhang, B., Yang, H., Peng, W., Liu, Q., et al. (2019). Structural and Functional Connectivity of the Anterior Cingulate Cortex in Patients With Borderline Personality Disorder.Frontiers in Neuroscience, 13 
28. Li, G., Zhang, W., Hu, Y., Wang, J., Li, J., Jia, Z., et al. (2021). Distinct Basal Brain Functional Activity and Connectivity in the Emotional-Arousal Network and Thalamus in Patients With Functional Constipation Associated With Anxiety and/or Depressive Disorders. Psychosomatic Medicine, 83(7), 707-714

29. Liu, L., Hu, C., Hu, Y., Zhang, W., Zhang, Z., Ding, Y., et al. (2021). Abnormalities in the thalamo-cortical network in patients with functional constipation. Brain Imaging and Behavior, 15(2), 630-642

30. Lorenz, J., Minoshima, S., \& Casey, K. L. (2003). Keeping pain out of mind: the role of the dorsolateral prefrontal cortex in pain modulation. Brain (London, England: 1878), 126(5), 1079-1091

31. Mayer, E. A., Aziz, Q., Coen, S., Kern, M., Labus, J. S., Lane, R., et al. (2009). Brain imaging approaches to the study of functional GI disorders: A Rome Working Team Report. Neurogastroenterology and motility, 21(6), 579-596

32. Mayer, E. A., Labus, J. S., Tillisch, K., Cole, S. W., \& Baldi, P. (2015). Towards a systems view of IBS. Nat Rev Gastroenterol Hepatol, 12(10), 592-605

33. Mugie, S. M., Benninga, M. A., \& Di Lorenzo, C. (2010). Epidemiology of constipation in children and adults: A systematic review. Baillière's best practice \& research. Clinical gastroenterology, 25(1), 3-18

34. Peters, S. K., Dunlop, K., \& Downar, J. (2016). Cortico-Striatal-Thalamic Loop Circuits of the Salience Network: A Central Pathway in Psychiatric Disease and Treatment. Front Syst Neurosci, 10, 104

35. Smith, S. M. (2002). Fast robust automated brain extraction. Human Brain Mapping, 17(3), 143-155

36. Starr, C. J., Sawaki, L., Wittenberg, G. F., Burdette, J. H., Oshiro, Y., Quevedo, A. S., et al. (2009). Roles of the Insular Cortex in the Modulation of Pain: Insights from Brain Lesions. Journal of Neuroscience, 29(9), 2684-2694

37. Stephani, C., Fernandez-Baca Vaca, G., Maciunas, R., Koubeissi, M., \& Lüders, H. O. (2011). Functional neuroanatomy of the insular lobe. Brain Structure and Function, 216(2), 137-149

38. Tamnes, C. K., Østby, Y., Fjell, A. M., Westlye, L. T., Due-Tønnessen, P., \& Walhovd, K. B. (2010). Brain Maturation in Adolescence and Young Adulthood: Regional Age-Related Changes in Cortical Thickness and White Matter Volume and Microstructure. Cerebral Cortex, 20(3), 534-548

39. Tillisch, K., Mayer, E. A., \& Labus, J. S. (2011). Quantitative Meta-analysis Identifies Brain Regions Activated During Rectal Distension in Irritable Bowel Syndrome. Gastroenterology, 140(1), 91-100

40. Tzourio-Mazoyer, N., Landeau, B., Papathanassiou, D., Crivello, F., Etard, O., Delcroix, N., et al. (2002). Automated anatomical labeling of activations in SPM using a macroscopic anatomical parcellation of the MNI MRI single-subject brain. Neuroimage, 15(1), 273-289

41. Uddin, L. Q. (2015). Salience processing and insular cortical function and dysfunction. Nature Reviews Neuroscience, 16(1), 55-61

42. Uddin, L. Q., Nomi, J. S., Hébert-Seropian, B., Ghaziri, J., \& Boucher, O. (2017). Structure and Function of the Human Insula. Journal of Clinical Neurophysiology, 34(4), 300-306

43. Wang, D., Zhang, X., Zhang, X., Huang, Z., \& Song, Y. (2017). Magnetic resonance imaging analysis of brain function in patients with irritable bowel syndrome.BMC Gastroenterology, 17(1) 
44. Wang, Y., Ji, G., Hu, Y., Li, G., Ding, Y., Hu, C., et al. (2020). Laparoscopic sleeve gastrectomy induces sustained changes in gray and white matter brain volumes and resting functional connectivity in obese patients. Surgery for Obesity and Related Diseases, 16(1), 1-9

45. Zhang, Z., Hu, Y., Lv, G., Wang, J., He, Y., Zhang, L., et al. (2020). Functional constipation is associated with alterations in thalamo-limbic/parietal structural connectivity. Neurogastroenterology \& Motility

46. Zhao, L., Wang, Y., \& Zhang, Y. (2018). Microstructural changes in the brain in elderly patients with irritable bowel syndrome. Aging Medicine, 1(2), 141-148

47. Zhu, Q., Cai, W., Zheng, J., Li, G., Meng, Q., Liu, Q., et al. (2016). Distinct resting-state brain activity in patients with functional constipation. Neuroscience Letters, 632, 141-146

48. ZUNG, W. W. K. (1965). Self-Rating Depression Scale in an Outpatient Clinic. Archives of General Psychiatry, 13(6), 508

49. Zung, W. W. (1971). A rating instrument for anxiety disorders. Psychosomatics, 12(6), 371-379

\section{Figures}

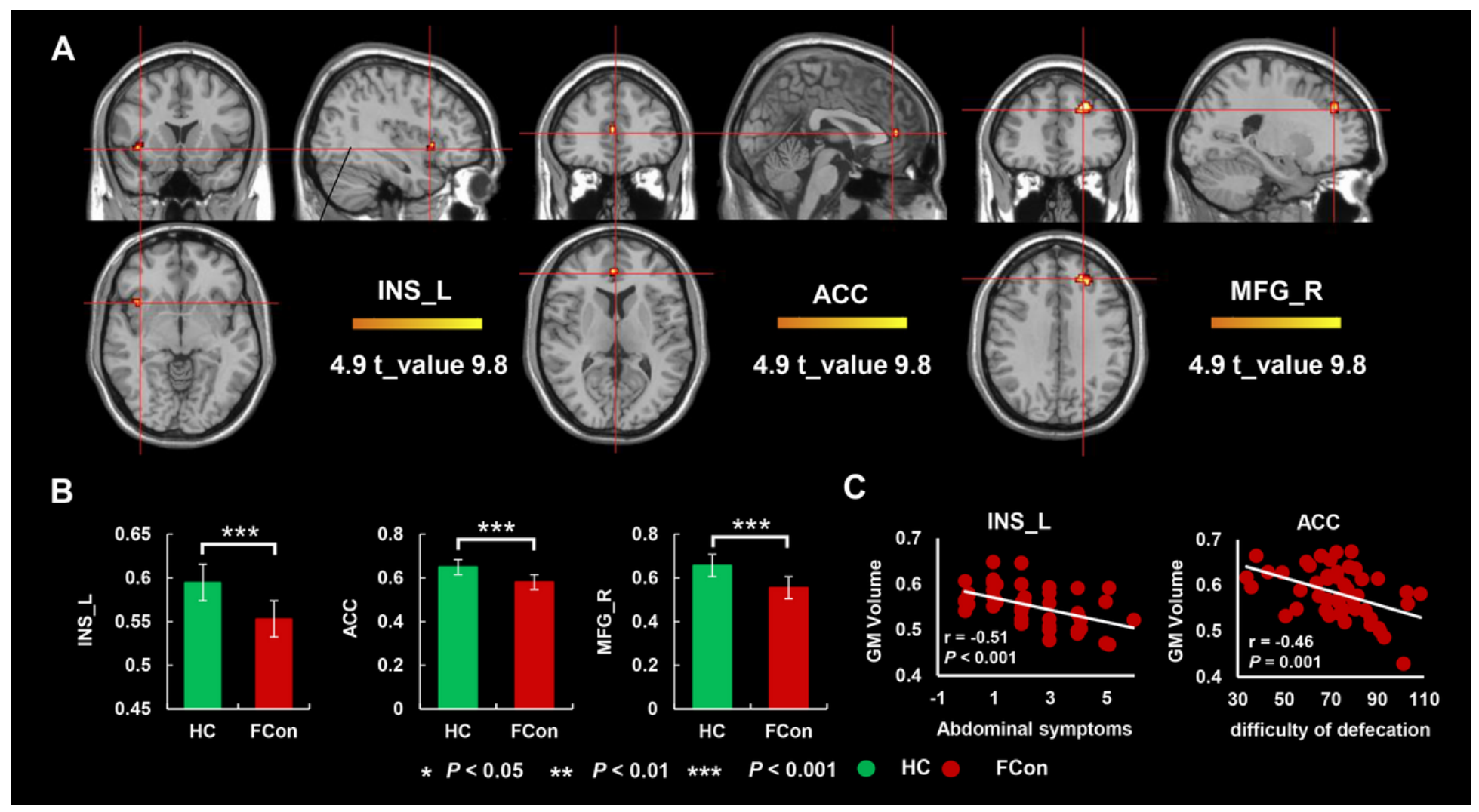

\section{Figure 1}

Two-sample t-test for GM volumes. (A and B) Compared with HC subjects, patients with FCon exhibited a decrease in GM volumes in the ACC, INS_L, and MFG_R (cluster size-corrected, PFWE < 0.05). Age and gender were regressed out as covariates. (C) Behavioral measurements had a significant negative correlation with GM volumes. Abbreviation: HC, healthy controls; FCon, functional constipation; ACC, 
anterior cingulate cortex; INS, insula; MFG, middle frontal gyrus; Error bars represent the standard error; L, left; $R$, right.

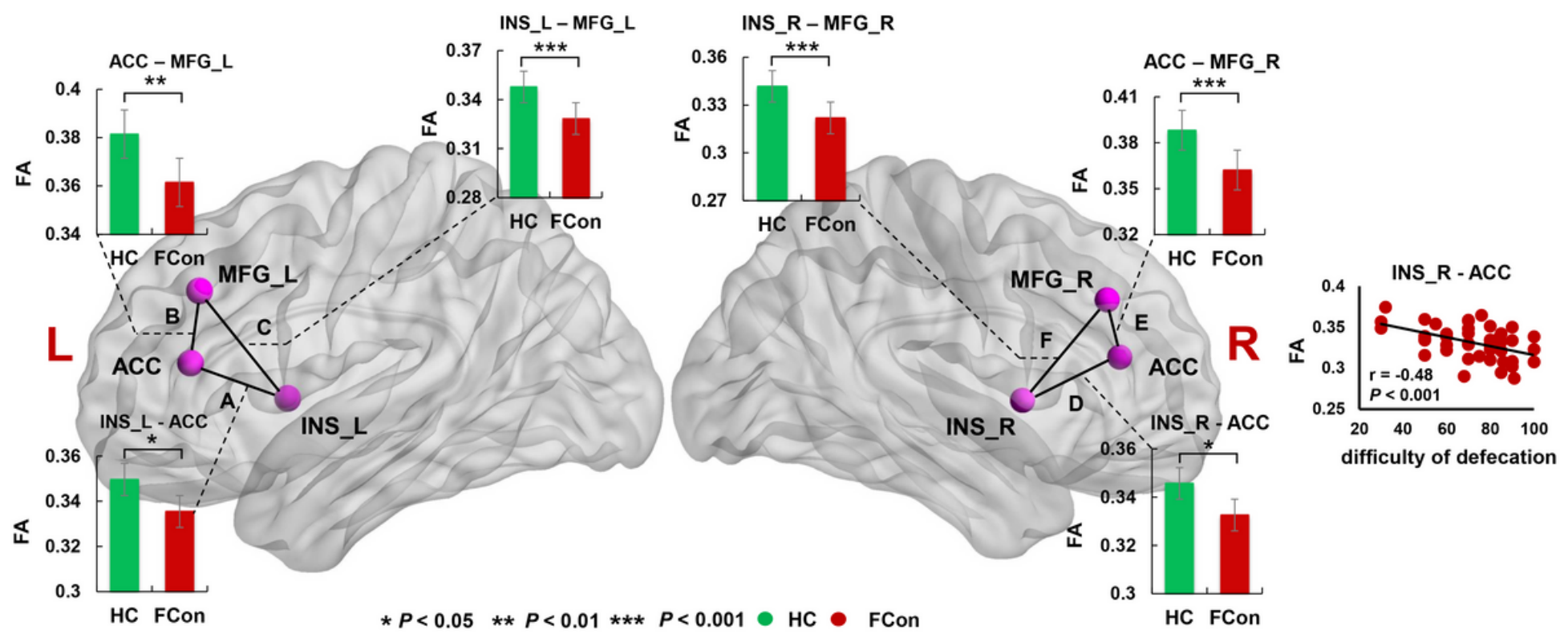

Figure 2

SC alterations in HC and FCon groups. FCon group showed significant decreased FA of the (A) left INS and $A C C,(B) A C C$ and left MFG, (C) left INS and left MFG, (D) right INS and ACC, (E) ACC and right MFG, and $(F)$ right INS and right MFG than $\mathrm{HC}$. Age and gender were regressed out as covariates. FA of the right INS and ACC tract had a negative correlation with difficulty of defecation. Abbreviation: HC, healthy controls; FCon, functional constipation; FA, fractional anisotropy; INS, insula; ACC, anterior cingulate cortex; MFG, middle frontal gyrus; Error bars indicate the standard error; L, left; $R$, right.

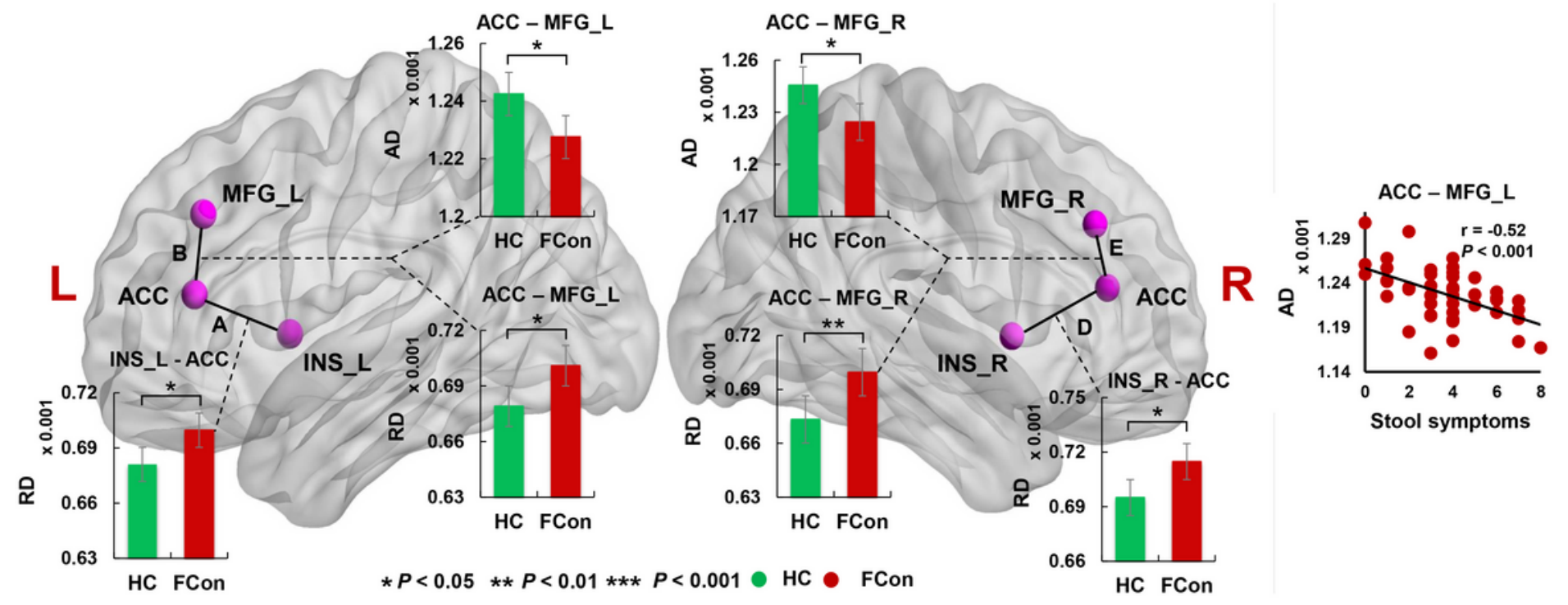

Figure 3 
FCon group showed significant increased RD of the (A) left INS and ACC, (B) ACC and left MFG, (D) right INS and ACC, and (E) ACC and right MFG than HC. FCon group showed significant decreased AD of (B) $A C C$ and left MFG and (E) ACC and right MFG than HC. Age and gender were regressed out as covariates. Stool symptoms had a negative correlation with AD of the ACC and left MFG tract. Abbreviation: $H C$, healthy controls; FCon, functional constipation; AD, axial diffusivity; RD, radial diffusivity; INS, insula; ACC, anterior cingulate cortex; MFG, middle frontal gyrus; Error bars indicate the standard error; L, left; R, right.

\section{Supplementary Files}

This is a list of supplementary files associated with this preprint. Click to download.

- BIBChecklist.docx 